\title{
Potential beneficial effects of ixmyelocel-T in the treatment of atherosclerotic diseases
}

\author{
Kelly J Ledford, Nikki Murphy, Frank Zeigler and Ronnda L Bartel
}

\begin{abstract}
Introduction: Advanced atherosclerotic lesions are characterized by lipid accumulation, inflammation, and defective efferocytosis. An ideal therapy should address all aspects of this multifactorial disease. Ixmyelocel-T therapy, an expanded autologous multicellular therapy showing clinical promise in the treatment of diseases associated with advanced atherosclerosis, includes a novel population of M2-like macrophages. Here, we examine the macrophages of ixmyelocel-T and determine their ability to influx modified cholesterol in an atheroprotective manner, maintaining cholesterol homeostasis and preventing cellular dysfunction and death, ultimately promoting reverse cholesterol efflux.
\end{abstract}

Methods: Approximately $50 \mathrm{ml}$ of whole bone marrow was obtained from healthy donors and shipped overnight. Bone marrow mononuclear cells (BMMNCs) were produced by using density gradient separation and cultured for approximately 12 days to generate ixmyelocel-T. CD14+ cells were isolated from ixmyelocel-T via positive selection for analysis. Ixmyelocel-T and human leukemia monocyte (THP-1) cells were loaded with acetylated low-density lipoprotein (Ac-LDL) for analysis. Flow cytometry and immunofluorescence were used to examine Ac-LDL uptake, expression of cytokines was analyzed by enzyme-linked immunofluorescence assay (ELISA), and quantitative realtime PCR was used to analyze expression of cholesterol-transport genes. Both the in vitro cholesterol efflux assay and in vivo reverse cholesterol transport assay were used to examine cholesterol transport.

Results: Ixmyelocel-T macrophages take up acetylated low-density lipoprotein and express the scavenger receptors CD36 and scavenger receptor-B1 (SR-B1). Ixmyelocel-T did not become apoptotic or proinflammatory after lipid loading. The cholesterol transporter genes $A B A C 1$ and $A B C G 1$ were both statistically significantly upregulated when ixmyelocel-T macrophages were loaded with cholesterol. Ixmyelocel-T also exhibited enhanced apolipoprotein A-I (ApoAI)-mediated cholesterol efflux. In addition, in vivo reverse cholesterol-transport assay demonstrated that ixmyelocel-T was able to efflux cholesterol in this model.

Conclusions: Ixmyelocel-T macrophages influx modified cholesterol, remained anti-inflammatory in the face of lipid loading and inflammatory challenge, and displayed enhanced cholesterol efflux capabilities. These combined features suggest that this autologous multicellular therapy may exert beneficial effects in atherosclerotic diseases.

\section{Introduction}

Atherosclerosis, a chronic inflammatory disease of the vessel wall, is a leading cause of death in developed countries [1]. Accumulation of lipid-loaded macrophage foam cells is a central feature in the formation of atherosclerosis. Macrophages, the most abundant cell type in atherosclerotic lesions, accumulate lipids, secrete inflammatory cytokines, and participate in all phases of atherosclerosis [2]. Maintaining macrophage cholesterol

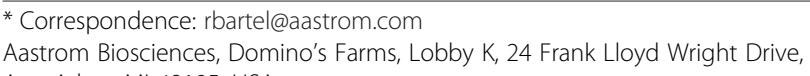

* Correspondence: rbartel@aastrom.com
Aastrom Biosciences, Domino's Farms, Lobby K, 24 Frank Lloyd Wright Drive, Ann Arbor, MI 48105, USA
}

homeostasis is essential in preventing the pathogenesis of atherosclerosis [3]. Macrophage-scavenger receptors, such as scavenger receptor (SR)-A and CD36, mediate the unregulated uptake of modified LDL lipoproteins, which leads to foam cell formation [3-5]. Cholesterol efflux, which removes excess cholesterol from macrophages, is believed to be the major process involved in the regression of atherosclerotic lesions [6,7]. The net flux, or influx and efflux, of modified cholesterol plays a key role in maintaining cholesterol homeostasis in macrophages [8]. An imbalance between cholesterol uptake by scavenger receptors and efflux in macrophages is widely 
recognized as the underlying mechanism leading to progression of atherosclerosis $[3,9]$.

Macrophage cholesterol efflux is considered to be protective against the development of atherosclerosis [10]. Free or unesterified cholesterol (FC) is toxic to macrophages and can lead to apoptosis [11]. To prevent cell death, cholesterol is stored as cholesterol esters (CEs) within macrophage foam cells and effluxed as FC. Cholesterol efflux is mediated by the ATP-binding cassette transporters $A B C A 1$ and ABCG1 [3]. ABCA1 regulates cholesterol efflux to lipid-free apolipoprotein A-I (apoA-I), and ABCG1 regulates cholesterol efflux to high-density lipoprotein (HDL) $[12,13]$. Both ABCA1 and ABCG1 prevent cholesterol accumulation and play an important role in maintaining macrophage cholesterol homeostasis. Excess ingested cholesterol is esterified by acyl-CoA:cholesterol acyl-transferase 1 (ACAT-1) and stored as lipid droplets in macrophages [3]. Cholesterol ester hydrolase (CEH) releases free cholesterol from stored $\mathrm{CE}$, allowing it to be removed from macrophages through cholesterol efflux [5]. Studies have shown that macrophages with high $\mathrm{CEH}$ activity accumulate fewer CEs. Promoting macrophage cholesterol efflux could prevent progression or induce regression of atherosclerosis [11].

Macrophages are a diverse population of cells that adapt and respond to a variety of signals, including cytokines and microbial products [14]. Classically activated macrophages, or M1, produce proinflammatory cytokines and kill microorganisms [14], whereas alternatively activated macrophages, or M2, regulate the inflammatory response by producing antiinflammatory cytokines, scavenging debris, and promoting tissue repair [14-18]. Both populations are considered extremes of a wide variety of macrophage phenotypes [19]. Atherosclerotic plaques have been found to contain a mixture of M1 and M2 macrophages [20,21]. Recent studies have suggested that M1 macrophages are dominant in both initial and developing atherosclerotic lesions, whereas M2 macrophages are associated with lesion regression $[20,22,23]$. It is thought that M2 macrophages may help prevent lesion progression by secreting antiinflammatory cytokines, such as interleukin (IL)-10, and efficiently removing apoptotic cells [20].

Ixmyelocel- $\mathrm{T}$ is an expanded autologous multicellular therapy containing a combination of cell types derived from cultured bone marrow mononuclear cells [24]. Ledford KJ, Murphy N, Zeigler F, Bartel RL (unpublished data) found that ixmyelocel-T contains a population of unique M2-like macrophages that are characterized by secretion of the antiinflammatory cytokines IL-10 and IL-1ra, with minimal secretion of the proinflammatory cytokines tumor necrosis factor-alpha (TNF- $\alpha$ ) and IL-12 after inflammatory challenge (Ledford KJ, Murphy N, Zeigler F, Bartel RL, unpublished data). Furthermore, these macrophages have demonstrated efferocytosis (that is, efficient removal of apoptotic cells) (Ledford KJ, et al., unpublished data). Both of these characteristics have been hypothesized to have beneficial effects in the treatment of atherosclerosis: IL-10 inhibits the secretion of inflammatory cytokines from other cells [20], and efferocytosis is considered atheroprotective [20]. Defective efferocytosis has been shown to promote atherosclerosis [25].

The goal of this investigation was to examine further ixmyelocel- $T$ macrophages and determine the ability of these cells to influx/efflux modified cholesterol in an atheroprotective manner, by maintaining cholesterol homeostasis and preventing cellular dysfunction and death, ultimately promoting reverse cholesterol efflux.

In this study, THP-1 macrophages were used as a control in these investigations. THP-1 cells are commonly used as a model to delineate macrophage gene expression and functions associated with modified cholesterol loading and have been established as an effective model to study the effects of cholesterol homeostasis [26-29].

\section{Methods}

\section{Cell culture}

For the generation of ixmyelocel-T, commercially available bone marrow aspirates (Lonza, Walkersville, MD, USA) were obtained from healthy donors under informed consent. A small amount (about $50 \mathrm{ml}$ ) of whole bone marrow was obtained through needle aspiration of the posterior iliac crest, and stored in heparinized tubes. The mononuclear cell fraction was obtained through an automated, closed-system, Ficoll-based density gradient centrifugation separation process. The isolated mononuclear cells were then transferred to a sterile, single-use cell cassette [30]. This proprietary system controlled temperature, culture-medium exchange, and gas exchange during the culture period. After approximately 12 days, the cells were washed and harvested from the cassette by a multistep, automated process. The cells were then used for experimental study.

THP-1 cells were obtained from ATCC (Manassas, VA, USA) and cultured in RPMI 1640 (ATCC) containing $10 \%$ fetal bovine serum and $2 \mathrm{~m} M$ L-glutamine, according to the manufacturer's instructions. THP-1 monocytes were differentiated into macrophages by using $200 \mathrm{n} M$ phorbol myristate acetate (InvivoGen, San Diego, CA, USA) for 3 days, after which they were used in the following experiments.

\section{CD $14^{+}$cell purification}

$\mathrm{CD}_{14}{ }^{+}$cells were isolated from ixmyelocel-T by positive selection by using MACS beads (Miltenyi Biotec, Bergisch Gladbach, Germany), according to the manufacturer's directions. After positive selection, the $\mathrm{CD} 14^{+}$ 
ixmyelocel-T macrophages were transferred to six-well culture plates for subsequent experiments.

\section{Dil-Ac-LDL immunostaining}

For fluorescent imaging, CD14 $4^{+}$ixmyelocel-T and THP-1 macrophages were plated in glass-chamber slides and allowed to adhere. Cells were loaded with $10 \mu \mathrm{g} / \mathrm{ml}$ DilAc-LDL in serum-free media containing $0.2 \%$ bovine serum albumin (BSA; fatty acid free; Sigma-Aldrich, St. Louis, MO, USA). After 24 hours, the cells were washed with phosphate-buffered saline (PBS) and fixed in 10\% formalin for 10 minutes. Counterstaining was performed with DAPI to visualize nuclei. Fluorescent images were visualized by using a Nikon Eclipse 80i (Nikon, Melville, NY, USA) equipped with an EXi Aqua Bio-Imaging Microscopy Camera (Q Imaging, Surrey, BC, Canada). For flow-cytometry quantification of ac-LDL uptake, ixmyelocel-T and THP-1 macrophages were loaded with $10 \mu \mathrm{g} / \mathrm{ml}$ Dil-Ac-LDL in serum-free media. DiIAcLDL uptake was analyzed with flow cytometry with at least 10,000 events acquired for each sample. DiIAcLDL uptake was quantified by mean fluorescent intensity, which was calculated by dividing the value of positive gated events by the value of the negative population.

\section{Cytokine secretion}

Enzyme-linked immunosorbent assay kits were used to determine the concentrations of IL-1 $\beta$, TNF- $\alpha$, IL-6, and monocyte chemoattractant protein (MCP)-1 (R\&D Systems, Minneapolis, MN, USA). In brief, CD14. ixmyelocel-T and THP-1 macrophages were loaded with $50 \mu \mathrm{g} / \mathrm{ml}$ acetylated low-density lipoprotein (Ac-LDL; Fisher Scientific, Pittsburg, PA, USA) for 24 hours in serum-free media containing 0.2\% BSA (fatty acid free). After 24 hours, the cells were washed with PBS and treated with lipopolysaccharide (LPS; $0.1 \mu \mathrm{g} / \mathrm{ml}$ ) overnight. Supernatants were then collected and assayed by following manufacturer's directions. For cytokine analysis, the values were normalized to cell number.

\section{Apoptosis assay}

Apoptosis was measured in both ixmyelocel-T and THP1 macrophages loaded with Ac-LDL. In brief, cells were seeded in 96-well plates and loaded with $100 \mu \mathrm{g} / \mathrm{ml}$ Ac-LDL for 24 hours in serum-free media containing $0.2 \%$ BSA (fatty acid free). The concentration of Ac-LDL used to induce apoptosis was based on our preliminary dose-finding study, which demonstrated that loading with $100 \mu \mathrm{g} / \mathrm{ml}$ Ac-LDL induced apoptosis in THP-1 macrophages. Induction of apoptosis was examined by measuring the caspase- 3 and caspase- 7 activity with the Caspase-Glo 3/7 Assay (Promega). Luminescence units were measured by using a SpectraMax plate reader (Molecular Devices, Sunnyvale, CA, USA), and values were expressed as a percentage relative to the values obtained from the control group (THP-1 macrophages).

\section{Cholesterol-efflux assay}

Ixmyelocel-T and THP-1 macrophages were plated at $0.5 \times 10^{6}$ cells/well, labeled with ${ }^{3} \mathrm{H}$-cholesterol for 24 hours, and allowed to equilibrate overnight. Cholesterol efflux was measured by using $20 \mu \mathrm{g} / \mathrm{ml}$ apoA-I and 20 $\mu \mathrm{g} / \mathrm{ml} \mathrm{HDL}_{3}$ in acceptor media after 4 hours. The efflux media and cells were analyzed to determine the percentage of cholesterol release.

\section{Reverse cholesterol transport}

Experiments were performed in severe combined immunodeficient (SCID) mice $(n=10)$. In brief, ${ }^{3} \mathrm{H}$-cholesterol-labeled ixmyelocel-T $(n=3)$ and J774 cells (3 to $5 \times 10^{6}$ ) $0.5 \mathrm{ml}$ MEM-HEPES were injected intraperitoneally. Blood was collected at 24 and 48 hours, and plasma was used for scintillation counting. Feces were collected continuously for 48 hours and used for scintillation counting.

\section{Real-time PCR}

Both CD14 ${ }^{+}$ixmyelocel-T and THP-1 macrophages were loaded with $50 \mu \mathrm{g} / \mathrm{ml}$ Ac-LDL for 24 hours in serumfree media containing $0.2 \%$ BSA (fatty acid-free). After 24 hours, the cells were washed with PBS and harvested for RNA isolation. For real-time PCR, total RNA was extracted with an RNeasy Mini kit (Qiagen, Valencia, CA, USA), and $1 \mu \mathrm{g}$ of RNA was reverse transcribed by using the high-capacity cDNA reverse transcription kit per the manufacturer's directions (Applied Biosciences, Carlsbad, CA, USA). Relative levels of target-gene expression were measured on the 7500 Real-Time PCR system (Applied Biosystems). FAM-based Taqman Gene Expression Assay Mix (Applied Biosystems) specific for each gene of interest and Taqman Universal Master Mix (Applied Biosystems) were used. Relative quantification PCR analysis was performed by using the ABI 7500 Software (Applied Biosystems). The relative amount of cDNA was calculated by normalization to GAPDH.

\section{Statistical analysis}

Paired $t$ tests were performed to compare results. A $P$ value less than 0.05 was considered statistically significant. Data are reported as mean \pm SEM.

\section{Results}

Ixmyelocel-T macrophages influx modified cholesterol

The ability to ingest modified cholesterol and the degree of Ac-LDL accumulation was examined with fluorescent microscopy and flow cytometry by using Dil-Ac-LDL (Figure 1). Fluorescent microscopy revealed that the THP-1 macrophages loaded with Dil-Ac-LDL appeared to be 


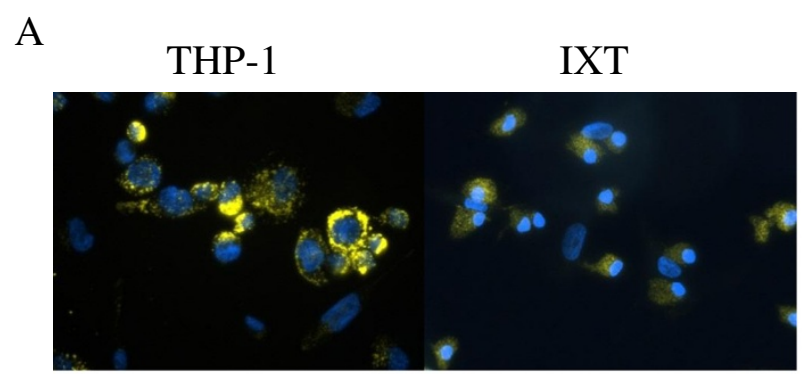

B

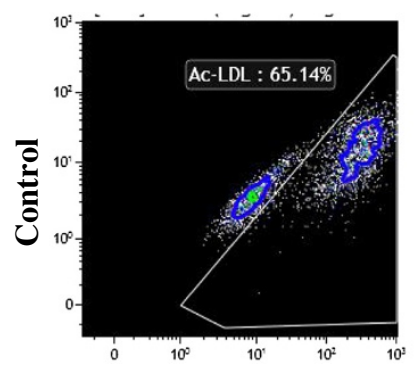

Ac-LDL

$\mathrm{C}$

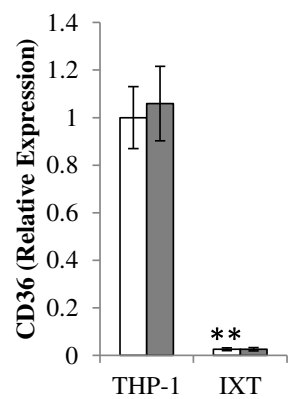

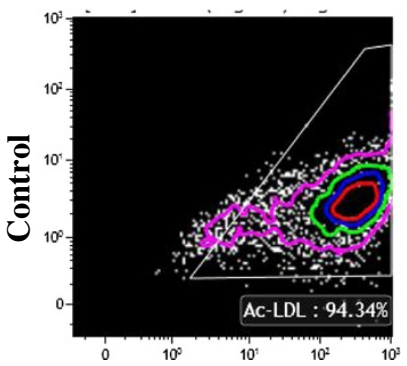

Ac-LDL

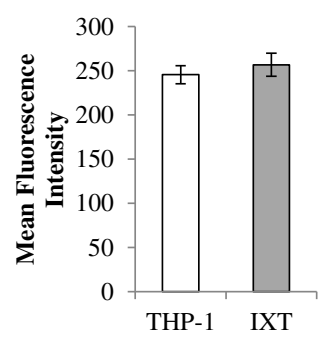

口-Ac-LDL

$\square+$ Ac-LDL

$\mathrm{D}$

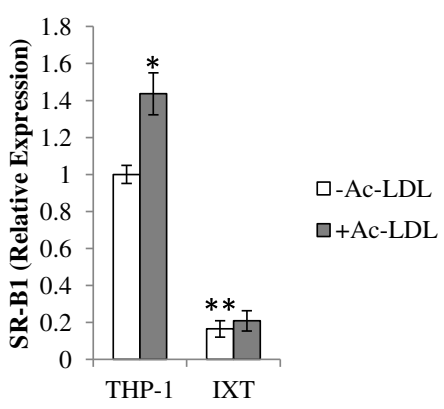

Figure 1 Ixmyelocel-T macrophages influx modified cholesterol. (A) Fluorescent microscopy images and (B) flow-cytometry analysis $(n \geq 5)$ of ixmyelocel-T and THP-1 macrophages loaded with Dil-Ac-LDL. Quantitative real-time PCR gene-expression analysis of scavenger receptors normalized to GAPDH ( $n \geq 5)$. Expression of (C) CD36 and (D) SCARB1 in THP-1 and ixmyelocel-T macrophages before and after lipid loading. Values are presented as mean \pm SEM relative to control; ${ }^{*} P<0.01$ versus THP-1 $-A C-L D L,{ }^{* *} P<0.001$ versus THP-1 $-A C-L D L$. Magnification: 40x. Dil-Ac-LDL, low-density lipoprotein from human plasma, acetylated, Dil complex; PCR, polymerase chain reaction; GAPDH, glyceraldehyde 3-phosphate dehydrogenase; SCARB1, scavenger receptor class B type I; SEM, standard error of the mean; Ac-LDL, acetylated low-density lipoprotein.

loaded with large lipid droplets (Figure 1A). Ixmyelocel-T macrophages loading with Dil-Ac-LDL appeared to be loaded with smaller lipid droplets (Figure 1A). Flow cytometry revealed that both ixmyelocel-T and THP-1 ingest similar amounts of Dil-Ac-LDL (257 \pm 13 versus $246 \pm 10$ mean fluorescent intensity; $P=0.26$; compared with THP-1; Figure 1B). These results provide evidence that ixmyelocel-T macrophages do influx modified cholesterol. These results also suggest that ixmyelocel-T macrophages do not store large droplets, or pools, of cholesterol.

To understand further the mechanisms of Ac-LDL uptake, real-time PCR was used to examine expression of the modified lipid-scavenger receptors CD36 and scavenger receptor (SR)-B1 before and after lipid loading with Ac-LDL. No statistically significant increase in CD36 expression was found after lipid loading in either ixmyelocel-T or THP-1 macrophages. THP-1 cells exhibited increased expression of $S R-B 1$ after lipid loading (1.44 \pm 0.11 versus $1 \pm 0.05$-fold change, $P<0.01$, compared with THP-1-Ac-LDL; Figure 1D), whereas no change in expression of $S R-B 1$ occurred in ixmyelocel-T macrophages treated with Ac-LDL $(0.16 \pm 0.04$ versus $0.21 \pm 0.05$-fold change; $P=$ NS compared with ixmyelocel$\mathrm{T}+$ Ac-LDL; Figure 1D). These data suggest that ixmyelocel-T macrophages influx Ac-LDL through scavenger receptors that ingest modified lipids. 


\section{Ixmyelocel-T does not become apoptotic after lipid loading}

To determine if lipid loading resulted in apoptosis, both ixmyelocel-T and THP-1 macrophages were loaded with $100 \mu \mathrm{g} / \mathrm{ml} \mathrm{Ac-LDL}$ for 24 hours. Apoptosis was then analyzed by using a luminometric caspase $3 / 7$ assay, which examines activation of caspases-3 and -7 . As shown in Figure 2, THP-1 macrophages exhibited a statistically significant higher level of Ac-LDL-induced apoptosis $(114 \% \pm 3.58 \%$ versus $100 \% \pm 3.58 \%$ of control; $P<0.01$ compared with THP-1 -Ac-LDL; Figure 2), than ixmyelocel-T $(87 \% \pm 10.78 \%$ versus $100 \% \pm 14.58 \%$ of control; $P=$ NS compared with ixmyelocel-T -Ac-LDL; Figure 2).

\section{Ixmyelocel-T secretes minimal amounts of} pro-inflammatory cytokines after lipid loading

When macrophages are unable to maintain cholesterol homeostasis because of ineffective cholesterol efflux, a proinflammatory response is generated [5]. Therefore, IL-1 $\beta$, TNF- $\alpha$, IL- 6 , and MCP-1 secretion was measured in lipid-loaded THP-1 and ixmyelocel-T macrophages before and after LPS stimulation (Table 1). When stimulated with LPS, THP-1 macrophages secreted significantly elevated amounts of IL- $1 \beta, \mathrm{TNF} \alpha$, IL-6, and MCP-1 (Table 1). When ixmyelocel-T macrophages were stimulated with LPS, a statistically significant increase in IL-6 was found; however, the level of IL-6 is lower compared with those from THP-1 macrophages (Table 1). Overall, THP-1 macrophages secreted significantly higher amounts of the inflammatory cytokines after LPS stimulation compared with ixmyelocel-T macrophages. THP-1 macrophages exhibited a 514-fold increase for IL-1 $\beta$, a 497-fold increase for TNF- $\alpha$, a 162-fold increase for IL-6, and a 32-fold increase for MCP-1 (Table 1). However, ixmyelocel- $\mathrm{T}$ macrophages exhibited a fivefold increase for IL-1 $\beta$, a twofold increase for TNF- $\alpha$, a threefold increase for IL-6, and a 1.5-fold increase for MCP-1 (Table 1). These data demonstrate that ixmyelocel-T

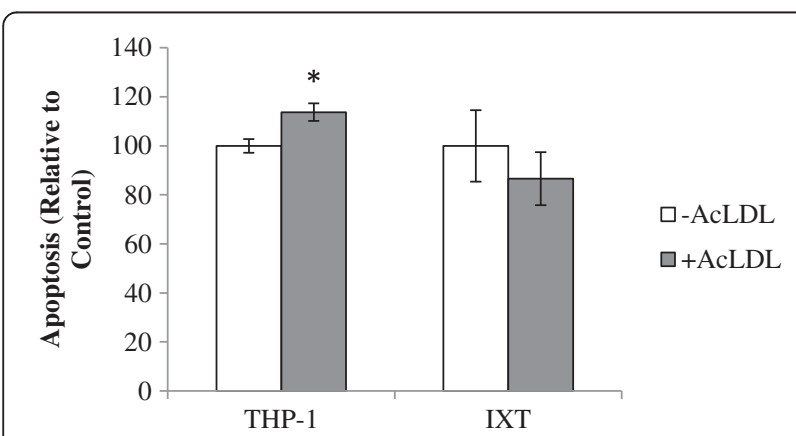

Figure 2 Ixmyelocel-T is not sensitive to modified cholesterolinduced lipotoxicity; percentage of apoptosis after cholesterol loading. Values are presented as mean \pm SEM relative to control; ${ }^{*} P<0.01$ versus THP-1 -AC-LDL. SEM, standard error of the mean; Ac-LDL, acetylated low-density lipoprotein.
Table 1 Ixmyelocel-T secretes minimal amounts of proinflammatory cytokines after lipid loading

\begin{tabular}{lllll}
\hline & THP-1 (-LPS) & THP-1 (+LPS) & \multicolumn{1}{c}{ IXT (-LPS) } & \multicolumn{1}{c}{ IXT (+LPS) } \\
\hline IL-1 $\beta$ & $25.7 \pm 6$ & $13,213 \pm 269^{\mathrm{a}}$ & $2.2 \pm 0.8^{\mathrm{b}}$ & $10 \pm 1.9^{\mathrm{a}}$ \\
TNF-a & $135 \pm 30$ & $67,063 \pm 8,539^{\mathrm{a}}$ & $86 \pm 54$ & $151 \pm 80^{\mathrm{a}}$ \\
IL-6 & $97 \pm 19$ & $15,733 \pm 1,874^{\mathrm{a}}$ & $769 \pm 353^{\mathrm{c}}$ & $2,376 \pm 551^{\mathrm{ad}}$ \\
MCP-1 & $940 \pm 120$ & $29,611 \pm 2,713^{\mathrm{a}}$ & $5,575 \pm 1,912^{\mathrm{c}}$ & $8,480 \pm 2,491^{\mathrm{a}}$ \\
\hline
\end{tabular}

Cytokines were quantified in supernatants from lipid-loaded ixmyelocel-T and THP-1 macrophages treated with and without LPS $(n \geq 6)$. The amount of cytokine expressed was normalized to the cell number of each sample. Values are presented as mean \pm SEM relative to control. ${ }^{a} P<0.001$ versus THP- 1 - LPS; ${ }^{b} P<0.01$ versus THP- 1 - LPS. ${ }^{C} P<0.05$, LPS, lipopolysaccharide; SEM, standard error of the mean; IXT, ixmyelocel-T. ${ }^{d} P<0.05$ versus IXT -LPS.

macrophages remain antiinflammatory even after lipid loading.

\section{Ixmyelocel-T displays enhanced cholesterol-efflux capacity}

With real-time PCR, expression of key genes involved in cholesterol efflux (for example, $A B C A 1$ and $A B C G 1$ ) was examined with and without cholesterol loading. There was a statistically significant increase in expression of $A B C A 1$ in ixmyelocel-T macrophages after cholesterol loading (1.49 \pm 0.32 versus $0.42 \pm 0.11$-fold change; $P<0.01$ compared with ixmyelocel-T -Ac-LDL; Figure $3 \mathrm{~A})$, whereas $A B C A 1$ was significantly decreased in THP-1 macrophages loaded with cholesterol $(0.78 \pm$ 0.05 versus $1 \pm 0.07$-fold change, $P<0.05$ compared with THP-1 -Ac-LDL; Figure 3A). Additionally, ixmyelocel-T macrophages expressed significantly more $A B C A 1$ after lipid loading compared with THP-1 macrophages $(1.49 \pm$ 0.32 versus $0.78 \pm 0.05$-fold change, $P<0.05$ compared with THP-1 + Ac-LDL; Figure 3A). Expression of ABCG1 was significantly increased in ixmyelocel- $T$ macrophages after cholesterol loading $(0.41 \pm 0.13$ versus $0.07 \pm 0.03$ fold change, $P<0.05$ compared with ixmyelocel-T -AcLDL; Figure $3 \mathrm{~B}$ ), whereas $A B C G 1$ was significantly decreased in THP-1 macrophages loaded with cholesterol $(0.75 \pm 0.04$ versus $1 \pm 0.04$-fold change, $P<0.001$ compared with THP-1 -Ac-LDL; Figure 3B).

$A C A T 1$ and $C E H$ were also examined in both cell populations. Ixmyelocel-T macrophages expressed significantly lower ACAT1 compared with THP-1 macrophages $(0.56 \pm 0.08$ versus onefold \pm 0.03 -fold change, $\mathrm{p}<0.001$ compared with THP-1 -Ac-LDL; Figure 3C). After lipid loading, no change was seen in ACAT1 expression in ixmyelocel- $T$ macrophages $(0.63 \pm 0.11$-fold versus $0.56- \pm 0.08$-fold change, $P=\mathrm{NS}$ compared with ixmyelocel-T -Ac-LDL; Figure 3C), whereas THP-1 macrophages expressed significantly elevated levels of ACAT1 (1.2-fold \pm 0.03 -fold versus onefold \pm 0.03 -fold change, $P<0.001$ compared with THP-1 -Ac-LDL; Figure 3C). After lipid loading, a statistically significant increase in $C E H$ expression was noted in ixmyelocel-T macrophages 

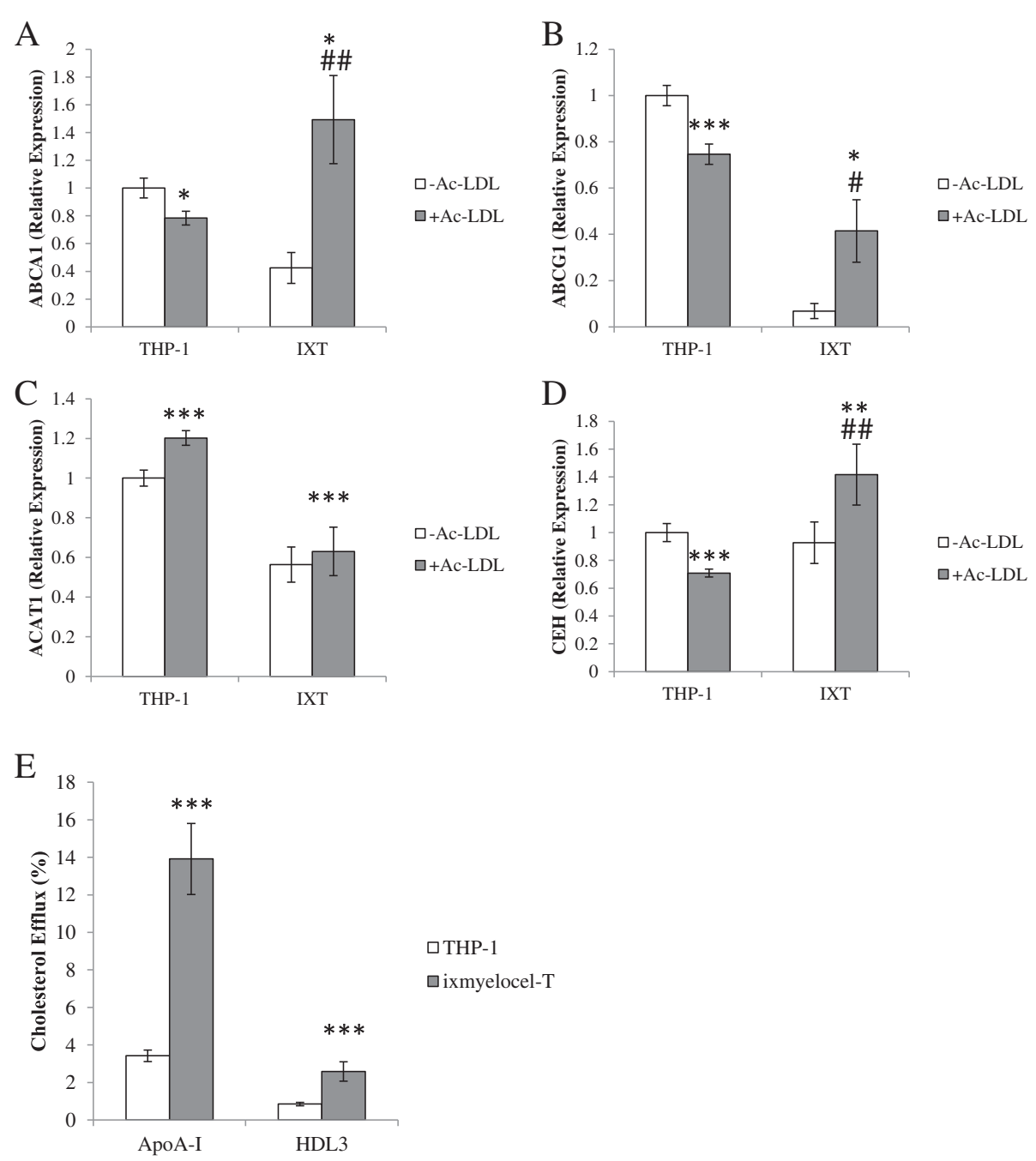

Figure 3 Ixmyelocel-T displays enhanced cholesterol-efflux capacity. Quantitative real-time PCR gene-expression analysis of scavenger receptors normalized to GAPDH ( $n \geq 5)$. Expression of (A) ABCA1, (B) ABCG1, (C) ACAT1, and (D) CEH in THP-1 and ixmyelocel-T macrophages before and after lipid loading. (E) Cholesterol efflux of both THP-1 macrophages and ixmyelocel-T to the lipid acceptors ApoA-I and HDL3 ( $n \geq 4$ ). Values are presented as mean \pm SEM relative to control; ${ }^{*} P<0.05,{ }^{* *} P<0.01,{ }^{* * *} P<0.001$ versus THP-1 -Ac-LDL; ${ }^{\#} P<0.05$, ${ }^{\# \#} P<0.01$ versus IXT -Ac-LDL. PCR, polymerase chain reaction; GAPDH, glyceraldehyde 3-phosphate dehydrogenase; ABC, ATP-binding cassette; ACAT, acyl-CoA:cholesterol acyl-transferase; CEH, cholesterol ester hydrolase; ApoA-I, apolipoprotein A-l; HDL, high-density lipoprotein; SEM, standard error of the mean; Ac-LDL, acetylated low-density lipoprotein.

(1.42 \pm 0.22 -fold versus $0.93 \pm 0.15$-fold change, $P<0.01$, compared with ixmyelocel-T -Ac-LDL; Figure 3D), whereas THP-1 macrophages expressed significantly decreased levels of $C E H(0.71 \pm 0.03$-fold versus 1 -fold \pm 0.06 -fold change, $P<0.001$ compared with THP-1 -AcLDL; Figure 3D). Because these genes, which are involved in cholesterol efflux, were upregulated in ixmyelocel- $T$, an in vitro cholesterol efflux assay was used to determine the ability of both cells to efflux cholesterol. Apolipoprotein A-I (ApoA-I)-mediated cholesterol efflux was significantly higher in ${ }^{3} \mathrm{H}$-cholesterol-AcLDL-loaded ixmyelocel-T compared with THP-1 macrophages $(13.91 \% \pm 1.90 \%$ versus $3.42 \% \pm 0.31 \%, P<0.001$ compared with THP-1;
Figure $3 \mathrm{E}$ ). $\mathrm{HDL}_{3}$-mediated cholesterol efflux was significantly higher in ${ }^{3} \mathrm{H}$-cholesterol-AcLDL-loaded ixmyelocel$\mathrm{T}$ compared with THP-1 macrophages $(2.58 \% \pm 0.052 \%$ versus $0.85 \% \pm 0.09 \%, P<0.01$ compared with THP-1; Figure 3E). Collectively, these data indicate that ixmyelocel-T macrophages exhibit enhanced capacities to efflux cholesterol.

\section{Ixmyelocel-T promotes reverse cholesterol transport}

The classic reverse cholesterol transport (RCT) pathway involves cholesterol efflux from macrophage foam cells to cholesterol acceptors and the excretion of cholesterol into the feces $[11,31]$. The in vivo RCT assay was used 
to determine whether ixmyelocel- $\mathrm{T}$ macrophages are capable of effluxing cholesterol in vivo. As shown in Figure 4, ixmyelocel- $\mathrm{T}$ is capable of promoting $\mathrm{RCT}$ in vivo. After injection of ${ }^{3} \mathrm{H}$-cholesterol-labeled ixmyelocel- $\mathrm{T}$ into the peritoneal cavity of SCID mice, plasma ${ }^{3} \mathrm{H}$-cholesterol levels were similar to the control cell line J774 cells after both 24 hours $(3.21 \% \pm 0.23 \%$ versus $3.48 \% \pm 0.39 \%$ injected cpm, $p=$ NS compared with J774; Figure $4 \mathrm{~A})$ and 48 hours $(2.92 \% \pm 0.22 \%$ versus $2.51 \% \pm$ $0.22 \%$ injected cpm, $P=\mathrm{NS}$ compared with J774; Figure 4A). Statistically significantly more ${ }^{3} \mathrm{H}$-tracer was found in the livers of mice injected with ixmyelocel-T compared with the J774 cells $(5.49 \% \pm 0.30 \%$ versus $3.79 \% \pm 0.39 \%$ injected $\mathrm{cpm}, P<0.05$ compared with 1774; Figure 4B), and no difference was found in the feces $(4.16 \% \pm 0.14 \%$ versus $4.85 \% \pm 0.57 \%$ injected cpm, $P=$ NS compared with J774; Figure 4C). These data demonstrate that ixmyelocel- $\mathrm{T}$ is able to efflux cholesterol in vivo, promoting cholesterol efflux in this model.

\section{Discussion}

The current study provides evidence that ixmyelocel-T macrophages influx and efflux modified cholesterol in an atheroprotective manner. The characteristics of
ixmyelocel-T macrophages help maintain cellular cholesterol homeostasis, preventing cellular dysfunction and death, and ultimately promoting reverse cholesterol efflux. These data point to a potential new innovative application of ixmyelocel- $T$ in the treatment of atherosclerotic diseases where massive amounts of modified cholesterol are present. Not only are ixmyelocel-T macrophages less susceptible to becoming foam cells, but they also appear to house the biologic machinery necessary to promote RCT, which has the potential to prevent progression or induce regression of atherosclerosis.

Uncontrolled accumulation of modified lipids in macrophages is a continuous process that contributes to all stages of atherosclerosis. Expression of scavenger receptors on macrophages is not downregulated by the accumulation of intracellular lipids, resulting in uncontrolled lipid accumulation and eventual irreversible cell damage and death [32]. This study and previously reported data (Ledford et al., unpublished data) demonstrated that ixmyelocel-T macrophages express relatively low levels of surface receptors involved in the uptake of modified cholesterol. Specifically, previous data demonstrated that ixmyelocel- $\mathrm{T}$ macrophages expressed lower levels of scavenger receptors involved in modified cholesterol uptake compared with M2 macrophages. In addition,
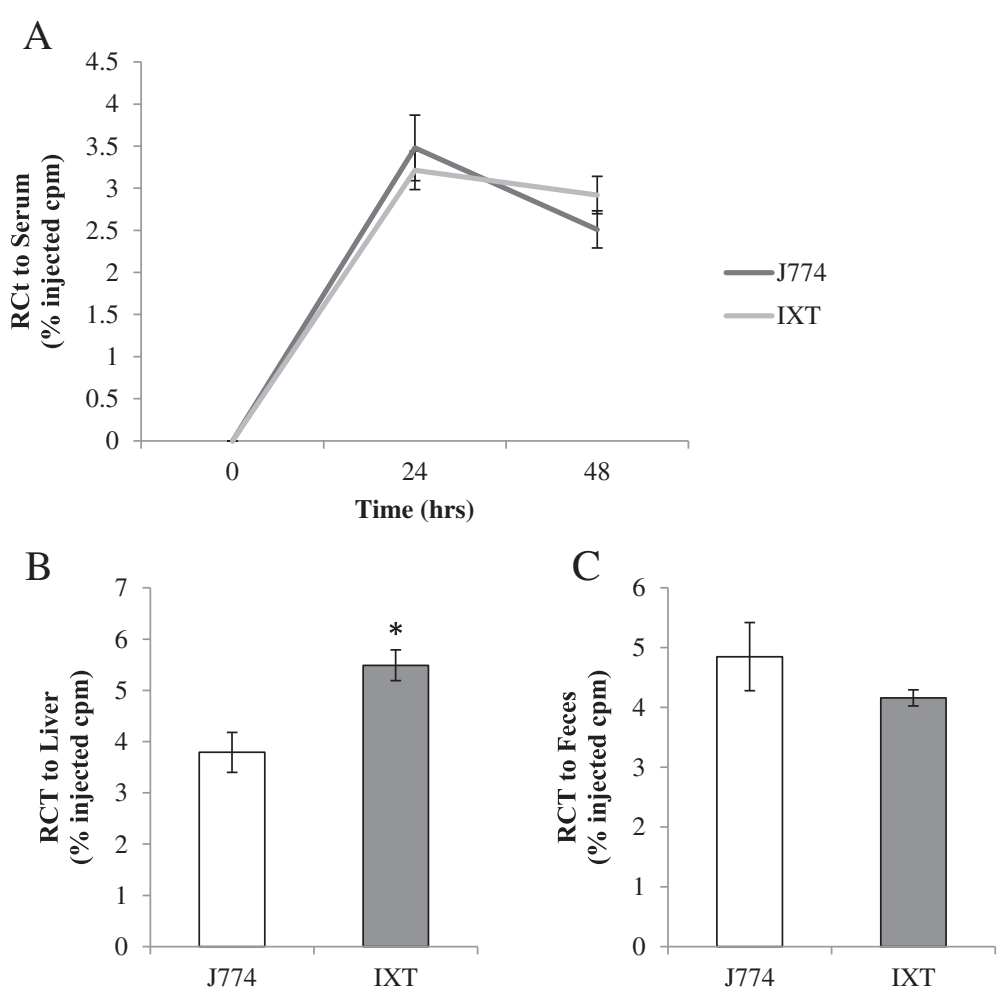

Figure 4 Ixmyelocel-T promotes reverse cholesterol transport. In vivo cholesterol efflux was examined in SCID mice after intraperitoneal injections of either ${ }^{3} \mathrm{H}$-cholesterol-loaded $\mathrm{J} 774$ cells or ixmyelocel-T. (A) Plasma ${ }^{3} \mathrm{H}$-cholesterol levels after 24 and 48 hours, (B) ${ }^{3} \mathrm{H}$-tracer found in the liver, and $(\mathbf{C}){ }^{3} \mathrm{H}$-tracer found in the feces after 48 hours ( $n \geq 3$ per group). Values are presented as mean \pm SEM relative to control, ${ }^{*} P<0.05$ versus J774. SCID, severe combined immunodeficient; SEM, standard error of the mean. 
ixmyelocel- $\mathrm{T}$ macrophages did not upregulate expression of these surface receptors in the presence of modified lipids. Fluorescence microscopy and flow cytometry demonstrated that ixmyelocel-T macrophages exhibited accumulation of modified LDL, showing that they do indeed influx modified cholesterol. However, fluorescence microscopy suggested that ixmyelocel- $T$ macrophages do not store large pools of cholesterol esters, suggesting that a majority of this cholesterol remains in the free state ready for efflux.

These data suggest that the macrophages generated by ixmyelocel-T may use protective mechanisms to prevent uncontrolled cholesterol loading, which is toxic to macrophages. These results suggest that ixmyelocel-T macrophages use a protective mechanism against unregulated modified cholesterol uptake by not upregulating scavenger receptor expression in the presence of modified cholesterol, as well as not storing vast amounts as esters. This observation is in alignment with the reduced apoptosis found in ixmyelocel- $T$ macrophages after cholesterol loading and the minimal increase in pro-inflammatory cytokine secretion after lipid loading and inflammatory challenge. The relatively low expression of scavenger receptors after modified cholesterol loading is one potential mechanism by which ixmyelocel-T is protected from cellular apoptosis after high lipid loading.

ACAT-1 promotes accumulation of intracellular CE in macrophages [33]. Studies have demonstrated that ACAT-1 plays an important role in atherosclerosis and is expressed at elevated levels in foam cells located within atherosclerotic lesions $[7,33]$. In addition, studies have also demonstrated that reduced ACAT-1 expression has been reported to inhibit foam cell formation in macrophages [33]. The results of this study show that a statistically significant decrease in ACAT-1 expression occurred in ixmyelocel-T macrophages when compared with THP-1 macrophages. Moreover, after loading with Ac-LDL, expression of $A C A T-1$ was not increased in ixmyelocel-T macrophages, a feature THP-1 macrophages did not share.

Hydrolysis of stored CE is the first and rate-limiting step in cholesterol efflux from macrophage foam cells, and increased expression of $\mathrm{CEH}$ leads to increased mobilization of $\mathrm{CE}$, thereby decreasing lipid accumulation [9]. After loading with modified cholesterol, ixmyelocel-T macrophages exhibited increased $C E H$ expression, the expression of which was decreased in THP-1 macrophages. These results may explain why the fluorescence microscopy images indicated that ixmyelocel-T macrophages do not store large pools of cholesterol esters. A decrease in ACAT-1 and increase in $C E H$ would suggest that a majority of the ingested cholesterol remains in the free state, ready for efflux. The combination of both increased $C E H$ and decreased ACAT-1 expression after cholesterol loading may be one mechanism by which ixmyelocel- $\mathrm{T}$ macrophages reduce cholesterol accumulation, thus preventing lipotoxicity.

Macrophages maintain cholesterol homeostasis with a balance between influx and efflux pathways [5]. To appreciate the potential impact of ixmyelocel-T macrophages on cholesterol homeostasis, it is essential to consider all aspects of cholesterol homeostasis, including efflux from cells. This investigation demonstrated that ixmyelocel-T macrophages display enhanced cholesterol efflux capacity. Specifically, these data demonstrated that after loading with Ac-LDL, ixmyelocel-T macrophages upregulated expression of both cholesterol transporter genes, $A B C A 1$ and $A B C G 1$. Both of these transporters are critical in the efflux of cholesterol from macrophages. In addition, results from the cholesterol efflux assay revealed that ixmyelocel- $\mathrm{T}$ macrophages were not only able to efflux cholesterol to the acceptor ApoAI and $\mathrm{HDL}^{3}$, but also to perform this action at an enhanced level. Ixmyelocel-T macrophages were also able to perform this action in vivo in the RCT assay.

Taken together, these data highlight the enhanced ability of ixmyelocel-T to promote RCT through cholesterol influx and efflux. In addition, the results highlight one mechanism by which ixmyelocel- $T$ macrophages maintain cholesterol homeostasis.

Atherosclerosis is chronic inflammatory disease that leads to a multitude of diseases, including coronary artery disease, peripheral artery disease, and stroke [34]. Several therapies have been investigated in atherosclerosis, including antiinflammatory drugs, cytokine, and chemokine antagonists. However, no really promising effects on clinical outcomes have been seen on the reduction of atherosclerosis in CAD [34]. Cell therapies have emerged for the treatment of cardiovascular diseases. Several studies have also provided evidence that cell therapies could be a useful treatment in of atherosclerosis, promoting lesion regression and thereby improving vascular health [34-36]. Furthermore, several studies have demonstrated that atherosclerotic lesions contain both M1 and M2 macrophages, providing evidence that M1 macrophages are found in unstable rupture-prone regions of atherosclerotic plaques [37-39]. Therefore, ixmyelocel- $T$ may be potentially beneficial in the treatment of atherosclerotic disease states in which they might promote lesion regression or stabilization through immunomodulation, efferocytosis, and modified cholesterol removal. Treatment with ixmyelocel-T has been investigated in bone regeneration, critical limb ischemia, and ischemic DCM. Further analysis of the subpopulations of cells generated in ixmyelocel-T could highlight new and innovative uses of this multicellular therapy. In this case, further analysis of ixmyelocel-T macrophages 
suggests that treatment with this cellular therapy might be advantageous in disease states involving atherosclerotic lesions, which would benefit from immunomodulation, efferocytosis, and modified cholesterol removal. Future studies will examine the effects of ixmyelocel-T in preclinical models to determine whether this therapy can affect the atherosclerotic state. For this cell therapy to affect an atheroma, it might need to be present in the lesion itself. Therefore, future studies will examine whether ixmyelocel-T has the ability to infiltrate atherosclerotic lesions or if it must be directly injected into areas where it might have the potential to dampen the immune response, remove necrotic debris, and efflux cholesterol.

\section{Conclusion}

The present data provide evidence that ixmyelocel- $T$ has the potential to become an effective adjuvant in the treatment of diseases due to atherosclerosis. Ixmyelocel-T contains a unique M2-like population of macrophages that are efficient at efferocytosis and maintaining cholesterol homeostasis. The data presented here suggest that ixmyelocel-T macrophages may exert beneficial effects in atherosclerotic disease because they influx cholesterol, remain antiinflammatory in the face of lipid loading and inflammatory challenge, and display enhanced cholesterol efflux capabilities. The multifactorial characteristics of the ixmyelocel-T M2-like macrophages described in this analysis, as well as the companion manuscript presented in Stem Cell Research and Therapy, highlight the potential effectiveness of this expanded autologous multicellular therapy in the treatment of atherosclerotic diseases.

\begin{abstract}
Abbreviations
ACAT: Acyl-CoA:cholesterol acyl-transferase; Ac-LDL: Acetylated low-density lipoprotein; apoA-l: Apolipoprotein A-l; BSA: Bovine serum albumin; CDNA: Complementary DNA; CEH: Cholesterol ester hydrolase; CES: Carboxylesterase; DAPI: 4'-6-diamidino-2-phenylindole; Dil-Ac-LDL: Low-density lipoprotein from human plasma acetylated, Dil complex; FAM: 6-carboxyyfluorescein; FBS: Fetal bovine serum; FC: Free or unesterified cholesterol; GAPDH: Glyceraldehyde 3-phosphate dehydrogenase; HDL: Highdensity lipoprotein; HEPES: 4-(2-hydroxyethyl) piperazine-1-ethanesulfonic acid; IL: Interleukin; LDL: Low-density lipoprotein; LPS: Lipopolysaccharide; MCP-1: Monocyte chemoattractant protein-1; MEM: Minimal essential medium; PBS: Phosphate-buffered saline; PCR: Polymerase chain reaction; $\mathrm{RCT}$ : Reverse cholesterol transport; RNA: Ribonucleic acid; RPMI: Roswell Park Memorial Institute medium; SCARB1: Scavenger receptor class B type I: SCID: Severe combined immunodeficient; SEM: Standard error of the mean; SR: Scavenger receptor; THP-1: Human leukemia monocyte; TNF-a: Tumor necrosis factor-alpha.
\end{abstract}

\section{Competing interests}

All authors are employees of Aastrom Biosciences, Inc.

\section{Authors' contributions}

$\mathrm{KL}$ conceived and designed research, acquired data, analyzed and interpreted data and results, performed statistical analysis, and drafted the manuscript. NM prepared samples, analyzed data, and reviewed and provided feedback during the development of the manuscript. FZ provided conceptual advice, analyzed data, and participated in discussion of results. RB contributed to the scientific direction, experimental approach, and interpretation of results. All authors read and approved the final manuscript.

\section{Acknowledgements}

The authors thank Alden Wong, Judith Schmitt, and Hillary Evens for excellent technical assistance. Scientific editorial support was sponsored by Aastrom Biosciences, Inc., and provided by David E. Kaminsky, PhD, of AlphaBioCom, LLC

Received: 19 March 2013 Revised: 26 August 2013

Accepted: 23 October 2013 Published: 1 November 2013

\section{References}

1. Yao S, Zong C, Zhang $Y$, Sang $H$, Yang $M$, Jiao $P$, Fang $Y$, Yang $N$, Song $G$, Qin S: Activating transcription factor 6 mediates oxidized LDL-induced cholesterol accumulation and apoptosis in macrophages by upregulating CHOP expression. J Atheroscler Thromb 2012, 20:94-107.

2. Hilgendorf I, Swirski FK: Making a difference: monocyte heterogeneity in cardiovascular disease. Curr Atheroscler Rep 2012, 14:450-459.

3. Chinetti-Gbaguidi G, Baron M, Bouhlel MA, Vanhoutte J, Copin C, Sebti Y, Derudas B, Mayi T, Bories G, Tailleux A, Haulon S, Zawadzki C, Jude B, Staels $B$ : Human atherosclerotic plaque alternative macrophages display low cholesterol handling but high phagocytosis because of distinct activities of the PPARgamma and LXRalpha pathways. Circ Res 2011, 108:985-995.

4. Pou J, Rebollo A, Roglans N, Sanchez RM, Vazquez-Carrera M, Laguna JC, Pedro-Botet J, Alegret M: Ritonavir increases CD36, ABCA1 and CYP27 expression in THP-1 macrophages. Exp Biol Med (Maywood) 2008, 233:1572-1582.

5. Ghosh S: Macrophage cholesterol homeostasis and metabolic diseases: critical role of cholesteryl ester mobilization. Exp Rev Cardiovasc Ther 2011, 9:329-340.

6. Moore KJ, Tabas I: Macrophages in the pathogenesis of atherosclerosis. Cell 2011, 145:341-355

7. Hongo S, Watanabe T, Arita S, Kanome T, Kageyama H, Shioda S, Miyazaki A: Leptin modulates ACAT1 expression and cholesterol efflux from human macrophages. Am J Physiol Endocrinol Metab 2009, 297:E474-E482.

8. Sankaranarayanan S, de la Llera-Moya M, Drazul-Schrader D, Asztalos BF, Weibel GL, Rothblat GH: Importance of macrophage cholesterol content on the flux of cholesterol mass. J Lipid Res 2010, 51:3243-3249.

9. Bie J, Zhao B, Ghosh S: Atherosclerotic lesion progression is attenuated by reconstitution with bone marrow from macrophage-specific cholesteryl ester hydrolase transgenic mice. Am J Physiol Regul Integr Comp Physiol 2011, 301:R967-R974.

10. Yamamoto S, Tanigawa H, Li X, Komaru Y, Billheimer JT, Rader DJ: Pharmacologic suppression of hepatic ATP-binding cassette transporter 1 activity in mice reduces high-density lipoprotein cholesterol levels but promotes reverse cholesterol transport. Circulation 2011, 124:1382-1390.

11. Cuchel M, Rader DJ: Macrophage reverse cholesterol transport: key to the regression of atherosclerosis? Circulation 2006, 113:2548-2555.

12. Suratt $B T$, Fessler MB: Greasing the way: the ABCs of HSPC efflux from the marrow. Cell Stem Cell 2012, 11:143-144.

13. Cuchel M, Lund-Katz S, de la Llera-Moya M, Millar JS, Chang D, Fuki I, Rothblat GH, Phillips MC, Rader DJ: Pathways by which reconstituted highdensity lipoprotein mobilizes free cholesterol from whole body and from macrophages. Arterioscler Thromb Vasc Biol 2010, 30:526-532.

14. Bouhlel MA, Derudas B, Rigamonti E, Dievart R, Brozek J, Haulon S, Zawadzki C, Jude B, Torpier G, Marx N, Staels B, Chinetti-Gbaguidi G: PPARgamma activation primes human monocytes into alternative $M 2$ macrophages with anti-inflammatory properties. Cell Metab 2007, 6:137-143.

15. Jennewein C, Kuhn AM, Schmidt MV, Meilladec-Jullig V, von Knethen A, Gonzalez FJ, Brune B: Sumoylation of peroxisome proliferator-activated receptor gamma by apoptotic cells prevents lipopolysaccharide-induced NCoR removal from kappaB binding sites mediating transrepression of proinflammatory cytokines. J Immunol 2008, 181:5646-5652.

16. Mosser DM: The many faces of macrophage activation. J Leukoc Biol 2003, 73:209-212.

17. Deonarine K, Panelli MC, Stashower ME, Jin P, Smith K, Slade HB, Norwood C, Wang E, Marincola FM, Stroncek DF: Gene expression profiling of cutaneous wound healing. J Trans/ Med 2007, 5:11

18. Mantovani A, Garlanda C, Locati M: Macrophage diversity and polarization in atherosclerosis: a question of balance. Arterioscler Thromb Vasc Biol 2009, 29:1419-1423.

19. Khallou-Laschet J, Varthaman A, Fornasa G, Compain C, Gaston AT, Clement M, Dussiot M, Levillain O, Graff-Dubois S, Nicoletti A, Caligiuri G: Macrophage plasticity in experimental atherosclerosis. PLoS One 2010, 5:e8852. 
20. Pello OM, Silvestre C, De Pizzol M, Andres V: A glimpse on the phenomenon of macrophage polarization during atherosclerosis. Immunobiology 2011, 216:1172-1176.

21. Hoeksema MA, Stoger JL, de Winther MP: Molecular pathways regulating macrophage polarization: implications for atherosclerosis. Curr Atheroscler Rep 2012, 14:254-263.

22. Cardilo-Reis L, Gruber S, Schreier SM, Drechsler M, Papac-Milicevic N, Weber C, Wagner O, Stangl H, Soehnlein O, Binder CJ: Interleukin-13 protects from atherosclerosis and modulates plaque composition by skewing the macrophage phenotype. EMBO Mol Med 2012, 4:1072-1086.

23. Feig JE, Vengrenyuk Y, Reiser V, Wu C, Statnikov A, Aliferis CF, Garabedian MJ, Fisher EA, Puig O: Regression of atherosclerosis is characterized by broad changes in the plaque macrophage transcriptome. PLoS One 2012, 7:e39790.

24. Bartel RL, Cramer C, Ledford K, Longcore A, Parrish C, Stern T, Watling S, Zeigler F: The Aastrom experience. Stem Cell Res Ther 2012, 3:26.

25. Thorp E, Tabas I: Mechanisms and consequences of efferocytosis in advanced atherosclerosis. J Leukoc Biol 2009, 86:1089-1095.

26. Auwerx J: The human leukemia cell line, THP-1: a multifaceted model for the study of monocyte-macrophage differentiation. Experientia 1991, 47:22-31.

27. Napolitano M, De Pascale C, Wheeler-Jones C, Botham KM, Bravo E: Effects of lycopene on the induction of foam cell formation by modified LDL. Am J Physiol Endocrinol Metab 2007, 293:E1820-E1827.

28. Huang Y, Ghosh MJ, Lopes-Virella MF: Transcriptional and posttranscriptional regulation of LDL receptor gene expression in PMAtreated THP-1 cells by LDL-containing immune complexes. J Lipid Res 1997, 38:110-120.

29. Michael DR, Salter RC, Ramji DP: TGF-beta inhibits the uptake of modified low density lipoprotein by human macrophages through a Smaddependent pathway: a dominant role for Smad-2. Biochim Biophys Acta 1822, 2012:1608-1616.

30. Yin D, Wang Z, Gao Q, Sundaresan R, Parrish C, Yang Q, Krebsbach PH, Lichtler AC, Rowe DW, Hock J, Liu P: Determination of the fate and contribution of ex vivo expanded human bone marrow stem and progenitor cells for bone formation by 2.3ColGFP. Mol Ther 2009, 17:1967-1978.

31. Annema W, Tietge UJ: Regulation of reverse cholesterol transport: a comprehensive appraisal of available animal studies. Nutr Metab (Lond) 2012, 9:25.

32. Rosenson-Schloss RS, Chnari E, Brieva TA, Dang A, Moghe PV: Glutathione preconditioning attenuates $\mathrm{AC}-\mathrm{LDL}$-induced macrophage apoptosis via protein kinase C-dependent Ac-LDL trafficking. Exp Biol Med (Maywood) 2005, 230:40-48.

33. Zhao ZZ, Wang Z, Li GH, Wang R, Tan JM, Cao X, Suo R, Jiang ZS: Hydrogen sulfide inhibits macrophage-derived foam cell formation. Exp Biol Med (Maywood) 2011, 236:169-176

34. Zenovich AG, Taylor DA: Atherosclerosis as a disease of failed endogenous repair. Front Biosci 2008, 13:3621-3636.

35. Lima LC, Porto ML, Campagnaro BP, Tonini CL, Nogueira BV, Pereira TM, Vasquez EC, Meyrelles SS: Mononuclear cell therapy reverts cuff-induced thrombosis in apolipoprotein E-deficient mice. Lipids Health Dis 2012, 11:96.

36. Porto ML, Lima LC, Pereira TM, Nogueira BV, Tonini CL, Campagnaro BP, Meyrelles SS, Vasquez EC: Mononuclear cell therapy attenuates atherosclerosis in apoE KO mice. Lipids Health Dis 2011, 10:155

37. Oh J, Riek AE, Weng S, Petty M, Kim D, Colonna M, Cella M, Bernal-Mizrachi C: Endoplasmic reticulum stress controls M2 macrophage differentiation and foam cell formation. J Biol Chem 2012, 287:11629-11641.

38. Shaikh S, Brittenden J, Lahiri R, Brown PA, Thies F, Wilson HM: Macrophage subtypes in symptomatic carotid artery and femoral artery plaques. Eur $\mathrm{J}$ Vasc Endovasc Surg 2012, 44:491-497.

39. Stoger IL, Gijbels MJ, van der Velden S, Manca M, van der Loos CM, Biessen EA, Daemen MJ, Lutgens E, de Winther MP: Distribution of macrophage polarization markers in human atherosclerosis. Atherosclerosis 2012, 225:461-468.

doi:10.1186/scrt346

Cite this article as: Ledford et al:: Potential beneficial effects of ixmyelocel-T in the treatment of atherosclerotic diseases. Stem Cell Research \& Therapy 2013 4:135.

\section{Submit your next manuscript to BioMed Central and take full advantage of:}

- Convenient online submission

- Thorough peer review

- No space constraints or color figure charges

- Immediate publication on acceptance

- Inclusion in PubMed, CAS, Scopus and Google Scholar

- Research which is freely available for redistribution 